\title{
Desquamating Nonpigmenting Fixed Drug Eruption With Onycholysis due to Amoxicillin in a Child: Cross-reactivity Study
}

\author{
Moya $\mathrm{B}^{1}$, Vera $\mathrm{A}^{1}$, Bazire $\mathrm{R}^{1,2}$, Betancor $\mathrm{D}^{1}$, Rodríguez Del \\ Río $\mathrm{P}^{1,2,3}$, Escudero $\mathrm{C}^{1,2,3}$, Ibáñez $\mathrm{MD}^{1,2,3}$ \\ ${ }^{1}$ Hospital Infantil Universitario Niño Jesús, Madrid, Spain \\ ${ }^{2} A R A D y A L$, FibHNJ, Madrid, Spain \\ ${ }^{3} I I S$-La Princesa, Madrid, Spain
}

J Investig Allergol Clin Immunol 2020; Vol. 30(2): 149-151 doi: 10.18176/jiaci.0466

Key words: Fixed drug eruption; Amoxicillin hypersensitivity in children. Drug allergy. Onycholysis. Nonpigmenting.

Palabras clave: Exantema fijo medicamentoso. Hipersensibilidad a amoxicilina en niños. Alergia a medicamentos. Onicolisis. No pigmentada.

Fixed drug eruption (FDE) is a delayed drug hypersensitivity reaction that rarely occurs in children. It typically appears as erythematous, well-circumscribed, and oval isolated macules that occur in isolation or in small numbers and cause intense pruritus, burning, or pain. They are most frequently located on the lips, palms, soles, glans penis, and groin and usually resolve spontaneously after discontinuation of the culprit drug, leaving hyperpigmentation [1]. They characteristically reappear at the same site if the patient is re-exposed to the drug. The drugs that most frequently cause FDE are analgesics, antimalarials, barbiturates, and antibiotics, including amoxicillin $[2,3]$. Diagnosis of FDE is based on skin tests and drug challenge tests (DCTs) [4]. There are rare clinical variants that include nonpigmenting FDE (NPFDE), generalized FDE, and other atypical presentations. NPFDE is a very rare variant that is characterized by the absence of residual hyperpigmentation. Few cases have been reported in adults [2], and only 2 cases have been reported in children $[5,6]$. We report a case of NPFDE with skin desquamation and onycholysis induced by amoxicillin, as confirmed with a DCT. Since studies assessing cross-reactivity in delayed type reactions in $\beta$-lactams are limited, we also evaluated cross-reactivity to other $\beta$-lactams by performing DCTs.

The patient was a 17-year-old male with Down syndrome and mild atopic dermatitis who had experienced 4 episodes of desquamation on the posterior aspect of the thumb and index finger of his right hand. The episodes had occurred 24-48 hours after finishing 7 days of oral amoxicillin treatment prescribed for infections such as tonsillitis. The first episode was at the age of 13 , and the patient has since experienced reactions to amoxicillin once annually. In one of the 4 episodes, the extension of the reaction also produced onycholysis on his right index finger that resolved spontaneously after a few weeks. In all the episodes, the skin lesions disappeared in 6-7 days without residual lesions. The patient previously tolerated penicillin, amoxicillin, and amoxicillin/clavulanic acid on several occasions. 
The allergological work-up was initiated by performing patch tests on the upper part of the patient's back with amoxicillin, amoxicillin/clavulanic acid, penicillin, and cefuroxime according to the guidelines of the European Society of Contact Dermatitis [7]. The patch test concentrations in petrolatum were as follows: amoxicillin, 50\%; amoxicillin/clavulanic acid, 50\% (amoxicillin/ clavulanic $875 / 125 \mathrm{mg}$ ); penicillin, $10 \%$; and cefuroxime, $10 \%$. All tests were negative at 48 and 96 hours. We also performed the tests with amoxicillin at the site of the lesion, although the patches detached quickly owing to the lack of adhesion on the fingers.

We performed a DCT with amoxicillin $750 \mathrm{mg}$ every 8 hours for 7 days. The first dose was administered in our department at 60-minute intervals (1/100, 1/10, full dose) [4], and the patient remained under medical surveillance for 2 hours. The remaining doses were taken at home. The patient presented desquamation starting on the posterior aspect of his thumb and index finger 2 days after finishing the final dose of amoxicillin (Figure). The lesions resolved spontaneously within 7 days, leaving no residual pigmentation.

Four months after the positive DCT with amoxicillin, we evaluated cross-reactivity to other ß-lactams with and without identical side chains using a DCT with penicillin $G$ and cefadroxil. The patient tolerated $750 \mathrm{mg}$ of penicillin $\mathrm{G}$ in our department (reached by gradual increases) followed by a dose of $750 \mathrm{mg}$ at home every 8 hours for 7 days. Two

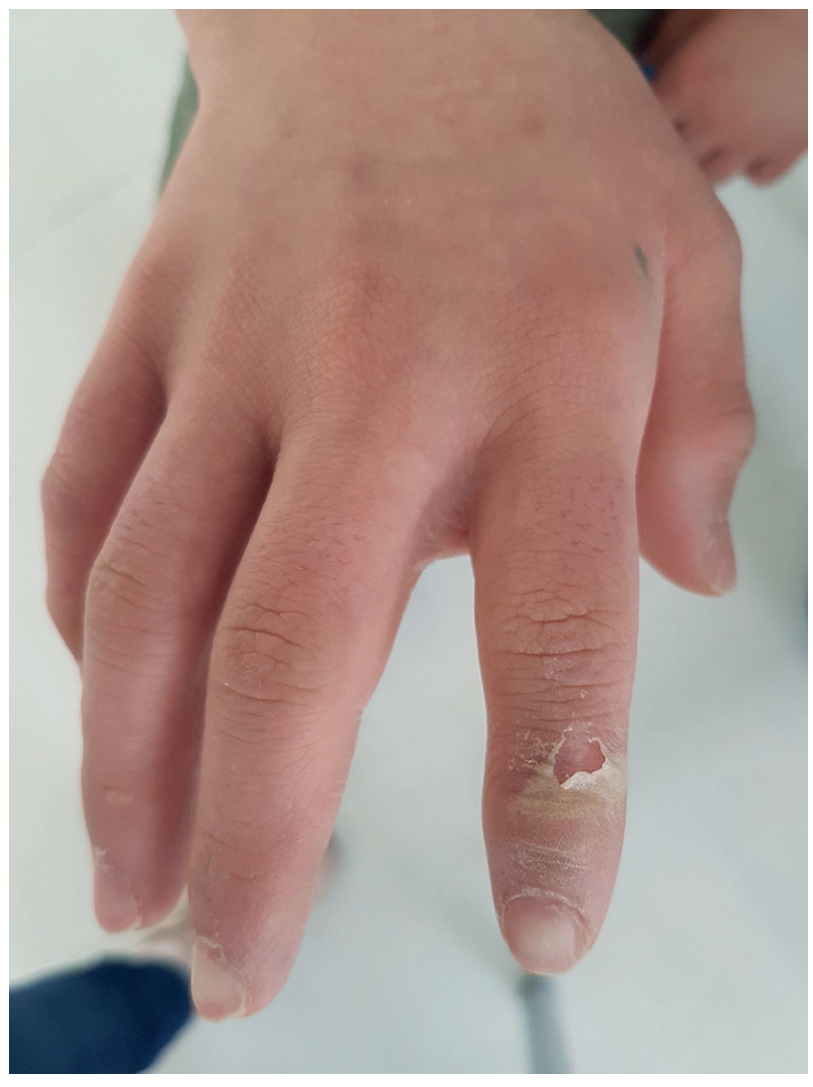

Figure. Desquamating nonpigmenting fixed drug eruption induced by the challenge test with amoxicillin. months later, a DCT performed with cefadroxil up to a total dose of $500 \mathrm{mg}$ under medical surveillance was negative. The following doses were administered at home every 12 hours. The patient experienced stomach discomfort after the first 2 doses, resulting in discontinuation of the treatment and inconclusive results for the evaluation of tolerance to cefadroxil. Outside the allergological study, the patient was also prescribed cefuroxime, a ß-lactam with a different side chain, which was also tolerated. Therefore, he was diagnosed with desquamative NPFDE induced by amoxicillin and advised not to take aminopenicillins or cephalosporins with the same side chain.

FDE is thought to be a classic delayed-type hypersensitivity reaction mediated by $\mathrm{CD}^{+}$cells. It has been demonstrated that intraepidermal $\mathrm{T}$ cells expressing the receptor TCR- $\alpha \beta$ are abundantly detected between keratinocytes in the lesions of FDE [1]. NPFDE is a rare delayed drug reaction. Only 2 cases have been reported in children: a 13-year-old with NPFDE in the form of bullous desquamation on the palms and soles induced by amoxicillin and rifamycin [5] and a 10-year-old boy with NPFDE in the form of penis swelling induced by amoxicillin [6]. To our knowledge, there have been no reported cases of desquamative NPFDE induced by amoxicillin, as demonstrated by DCT, in a boy.

While better methods for diagnosing FDE continue to be debated, DCT is the most reliable approach for identifying the causative drug [1]. In this case, a positive DCT result with amoxicillin confirmed the diagnosis in a patient with negative patch test results. The use of patch tests has been controversial owing to the elevated number of false-negative results [1].

While cross-reactivity between ß-lactams in type I reactions (immediate) has been evaluated more extensively [8], it has received less attention in non-type I reactions (delayed) [9]. Cross-reactivity may occur because amoxicillin and some early-generation cephalosporins share identical side chains (eg, cefadroxil, cefatrizine, and cefprozil) or similar side chains (eg, cefamandole and cefonicid) [10]. Further research is still needed to evaluate cross-reactivity in FDE caused by ß-lactams.

By studying cross-reactivity, we demonstrated the aminoselectivity of the reaction and provided the patient with safer, alternative $\beta$-lactams.

In conclusion, we present the first case of NPDFE with skin desquamation and onycholysis caused exclusively by amoxicillin in a teenage patient who tolerated other ß-lactams with a different side chain. DCT is currently the most reliable method for the identification of the drug implicated in FDE. An allergological work-up makes it possible to provide safe alternative $\beta$-lactams, although more research is needed to assess cross-reactivity in delayed reactions caused by ß-lactams.

\section{Funding}

The authors declare that no funding was received for the present study.

\section{Conflicts of Interest}

The authors declare that they have no conflicts of interest. 


\section{References}

1. Shiohara T. Fixed drug eruption: pathogenesis and diagnostic tests. Curr Opin Allergy Clin Immunol. 2009;9:316-21.

2. Mahboob A, Haroon TS. Drugs causing fixed eruptions: a study of 450 cases. Int J Dermatol. 1998;37:833-8.

3. Galindo PA, Borja J, Feo F, Gómez E, Encinas C, García R. Nonpigmented fixed drug eruption caused by paracetamol. J Investig Allergol Clin Immunol. 1999;9(6):399-400.

4. Labrosse R, Graham F, Bégin P, Samaan K, Paradis L, Des Roches $A$, et al. A pediatric case of selective fixed drug eruption to amoxicillin. Pediatr Allergy Immunol. 2017 Dec;28(8):848-50.

5. Ponvert $C$, Rufin $P$, de Blic J. An unusual case of nonpigmenting fixed drug eruptions in a child. Pediatr Allergy Immunol. 2013;24:715-6.

6. Keli-Bhija Z; De Blic J. Ponvert C. An unusual case of penile non-pigmenting fixed drug eruption in a child. Pediatr Allergy Immunol. 2015;26:294-6.

7. Johansen JD, Aalto-Korte $K$, Agner $T$, Andersen KE, Bircher A, Bruze $M$, et al. European society of contact dermatitis guideline for diagnostic patch testing - recommendations on best practice. Contact Dermatitis. 2015;73:195-221.

8. Romano A, Valluzzi RL, Caruso C, Maggioletti M, Quaratino D, Gaeta F, et al. Cross-Reactivity and Tolerability of Cephalosporins in Patients with IgE-Mediated Hypersensitivity to Penicillins. J Allergy Clin Immunol Pract. 2018;6:1662-72.

9. Buonomo, A., Nucera, E., Pecora, Rizzi A, Aruanno A, Pascolini $L$, et al. Cross-reactivity and tolerability of cephalosporins in patients with cell-mediated allergy to penicillins. J Investig Allergol Clin Immunol. 2014;24(5):331-7.

10. Zagursky RJ, Pichichero ME. Cross-reactivity in $\beta$-Lactam Allergy. J Allergy Clin Immunol Pract. 2018;6:72-81.

I Manuscript received July 7, 2019; accepted for publication November 19, 2019.

María Dolores Ibáñez Sandín Allergy Department Hospital Infantil Universitario Niño Jesús Avda/ Menéndez Pelayo, No 65 28009 Madrid, Spain

E-mail: mibanezs@salud.madrid.org 